\title{
Medication adherence and the risk of severe asthma exacerbations: a systematic review
}

\author{
Marjolein Engelkes ${ }^{1}$, Hettie M. Janssens ${ }^{2}$, Johan C. de Jongste ${ }^{2}$, \\ Miriam C.J.M. Sturkenboom ${ }^{1}$ and Katia M.C. Verhamme ${ }^{1}$
}

\author{
Affiliations: \\ ${ }^{1}$ Dept of Medical Informatics, Erasmus University, Rotterdam, The Netherlands. \\ ${ }^{2}$ Dept of Pediatrics/Respiratory Medicine, Erasmus University/Sophia Children's Hospital, Rotterdam, The \\ Netherlands. \\ Correspondence: Marjolein Engelkes, Erasmus MC, Medical Informatics, P.O. Box 2040, Rotterdam 3015 CA, \\ The Netherlands. E-mail: m.engelkesderasmusmc.nl
}

ABSTRACT The benefits of drug therapy for asthma have been well established, but adherence to treatment is poor, and this might be associated with an increased risk of asthma exacerbations. The aim of this study was to review the literature on the association between adherence to asthma controller treatment and risk of severe asthma exacerbations in children and adults.

A systematic literature search was performed in PubMed, Embase and Web of Science, from inception until January 2014. Studies were included if data on the association between medication adherence and severe asthma exacerbations were presented. Quality was assessed using a modified version of the Newcastle-Ottawa Scale.

The search yielded 2319 unique publications, of which 23 met the inclusion criteria and underwent data extraction and quality scoring. High levels of heterogeneity across studies with regard to adherence and exacerbation measurements, designs and analysis precluded a formal meta-analysis. Although effect measures varied widely, good adherence was associated with fewer severe asthma exacerbations in highquality studies.

Good adherence tended to be associated with lower risk of severe asthma exacerbations. Future studies should use standardised methodology to assess adherence and exacerbations, and should consider inhaler competence.

@ERSpublications

High-quality studies indicated that better adherence is associated with fewer severe asthma exacerbations http://ow.ly/BU6ro

\footnotetext{
Earn CME accreditation by answering questions about this article. You will find these at erj.ersjournals.com/misc/ cmeinfo.xtml
}

This article has supplementary material available from erj.ersjournals.com

Received: Nov 252013 | Accepted after revision: Aug 272014 | First published online: Oct 162014

Conflict of interest: Disclosures can be found alongside the online version of this article at erj.ersjournals.com

Copyright CERS 2015 


\section{Introduction}

Asthma is a chronic inflammatory airway disease with a high prevalence, around $10 \%$ in children and $5 \%$ in adults in Western countries $[1,2]$. Asthma is a major cause of disability and health resource utilisation, and reduces quality of life [3]. This is partly caused by asthma exacerbations, which have a huge impact on patients and their families. To minimise asthma exacerbations, treatment should be adjusted stepwise, driven by the patient's asthma control level [4]. Asthma treatment includes daily use of a controller drug and use of short-acting bronchodilators when needed for quick symptom relief [5]. Adherence to treatment is essential to optimise the benefits of therapy. Poor adherence has been associated with outcomes like mortality [6], asthma symptoms [7], direct and indirect costs of care [3] and quality of life [8]. In asthma, adherence to treatment tends to be poor, with rates of $<50 \%$ in children [9] and $30-70 \%$ in adults $[4,10,11]$, depending on country, age, sex and ethnicity [12]. These low adherence rates have been attributed to safety concerns about inhaled corticosteroids (ICS) ("steroid phobia") by both the patients and the caregivers [13]. Indeed, use of ICS has been associated with growth impairment in children and other systemic adverse effects, such as an increased risk of pneumonia [14]. In addition, most ICS need to be administered twice daily, which increases the risk of poor adherence compared with once-daily administration [5]. It has been suggested that poor adherence to ICS increases the risk of exacerbations. However, the literature on this topic is conflicting. With this systematic review, we aim to provide a critical appraisal of the literature, examining the association between adherence to asthma controller therapy and the risk of severe asthma exacerbations in children and adults.

\section{Methods}

Electronic searches

An extensive electronic literature search was conducted to identify all relevant articles, published from inception up to January 1, 2014, as indexed by PubMed, Web of Science and Embase (online supplementary table S1). Article reference lists were searched for additional potentially relevant articles.

\section{Review criteria and data extraction}

All original articles were considered, excluding case reports, audits, guidelines, editorials, management/ implementation strategies, conference abstracts and study protocols. We excluded animal studies. No limits were set on study design, sample size, location or follow-up. Eligible patient populations included both children and adults using asthma controller therapy: ICS, long-acting $\beta_{2}$-agonists (LABAs) or fixed combination therapies of LABA and ICS. Due to incomparability, we excluded studies that looked at leukotriene receptor antagonists or xanthines only.

Studies were included if they met the following three criteria. 1) The exposure variable of interest was medication adherence to asthma controller therapy. Medication adherence is an umbrella term that encompasses the concepts of compliance and persistence. Compliance is defined as the extent to which a patient acts in accordance with the prescribed interval and dosing regimen, and persistence is defined as the duration of time from initiation to discontinuation of therapy, according to the International Society for Pharmacoeconomics and Outcomes Research [15]. Only studies with objectively measured adherence, including electronic monitoring devices, pill counting and prescription/refill data were included. Adherence measured through subjective measures, such as patient self-reports, questionnaires and physician's judgments were excluded as we considered these to be less reliable and not comparable to objective adherence measures. 2) The outcome of interest was severe asthma exacerbation. According to the joint Global Initiative for Asthma [4], American Thoracic Society [16] and European Respiratory Society [17] asthma guidelines, this outcome was defined as "the occurrence of either an asthma-related hospitalisation or visit to the emergency department or an urgent care facility, leading to treatment with systemic (oral, intramuscular or intravenous) corticosteroids for at least 3 days". 3) Evaluation of the association between adherence and exacerbations as primary or secondary end-point.

The first author (M. Engelkes) assessed the eligibility of studies from their titles and abstracts, excluding those that were not relevant. The full texts of eligible papers were assessed independently for eligibility by two authors (M. Engelkes and K.M.C. Verhamme) and data were extracted into a customised data extraction Excel form. Third party adjudication was foreseen in case of disagreement.

\section{Assessment of methodological quality}

All included studies underwent a formal evaluation according to the Newcastle-Ottawa Scale, a set of criteria established and used in previous systematic reviews of observational studies [18, 19], which was modified for the purpose of this review. All studies were independently rated by two reviewers (M. Engelkes and K.M.C. Verhamme), to assess the quality based on five parameters: overall design, selection of participants, exposure assessment, outcome ascertainment and control for extraneous factors. 
Each parameter received 0,1 or 2 points (online supplementary table S2). The total score represented the sum of all five parameters. This score was used as a relative measure of data quality.

\section{Data analysis}

Pooling of studies was considered in case of adequate similarity, with respect to exacerbation definitions, adherence definitions and methods to assess the relationship between adherence and exacerbations. In case of heterogeneity, the results of each study were reported individually.

\section{Results}

\section{Overview of the included studies}

The search strategy identified 2319 articles. Upon title and abstract review, 2268 of these were excluded, mainly for the following reasons: lack of information on medication adherence, no severe asthma exacerbation or no evaluation of the relationship between adherence and exacerbation $(n>1000)$; articles on intervention, management or implementation strategies $(n=370)$; audit/guidelines $(n=122)$; focus on other diseases $(n=139)$; animal studies $(n=106)$; cell biology/immunology $(n=62)$; not original articles $(n=40)$. A total of 23 articles were finally included in the review (fig. 1).

Details of the 23 studies are shown in table 1. Briefly, all 23 studies were published between 1993 and 2013. The sample sizes ranged from 24 to 97743 individuals $[9,33]$. Two studies were multicentre, seven were single-centre and 14 were based on healthcare (pharmacy/insurance/claim) databases. Most studies were from the USA. The included studies mainly used a cohort design $(n=19)$, with the remainder utilising a cross-sectional $(n=1)$ [9], case-control $(n=1)$ [30] or randomised design $(n=2)[24,40]$. Studies analysed adherence rates over a follow-up period ranging from 13 weeks [9] to 4 years [40]. 10 studies included only children, seven studies included both adults and children/adolescents, and six studies included only adults.

\section{Measures of medication adherence}

The assessment of medication adherence varied across studies. Most studies $(n=11)$ used the medication possession rate (MPR) as a measure, which is the number of days of medication supplied divided by the number of days between the first and the last refill [43]. Most studies chose a fixed time-frame for the refill interval rather than using the last refill as the end-point for the refill interval, and did not exclude the last refill. MPR calculated across multiple refills is also called the continuous measure of availability. In addition to the MPR, two studies also calculated a controller-to-total ratio [21, 27]. WilLiAMs et al. [28] used a unique method to calculate adherence, by calculating a moving 6-month average ICS adherence for each day of follow-up. Furthermore, six studies used number of (requested) refills in a certain period to

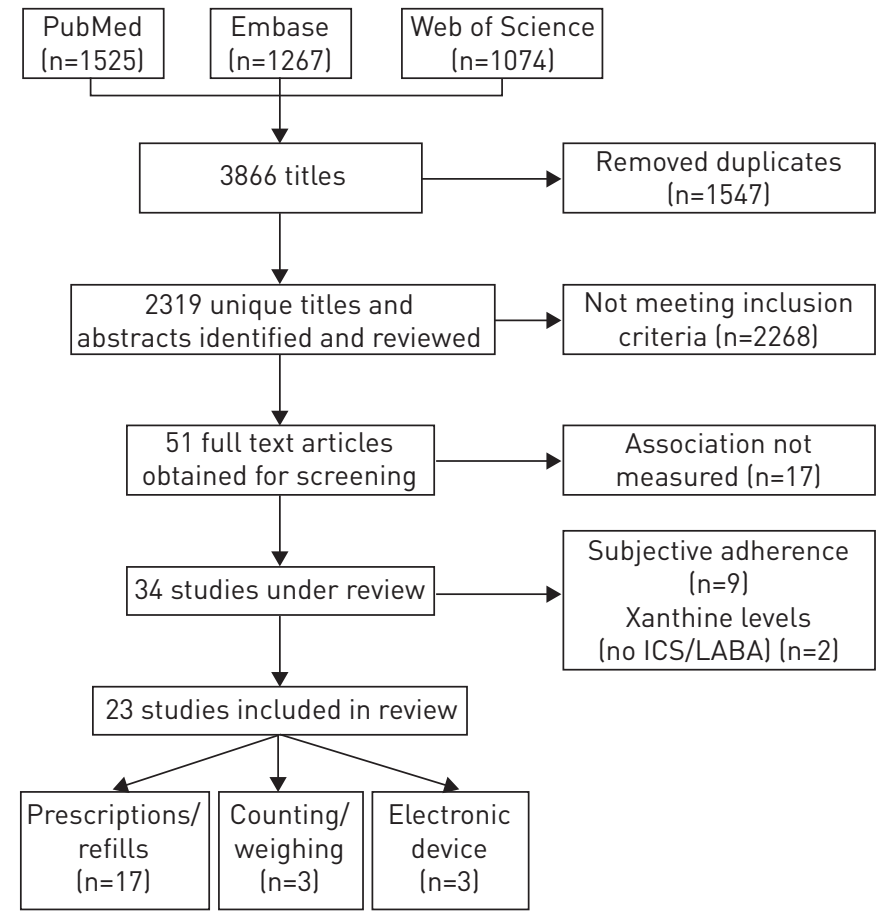

FIGURE 1 Preferred Reporting Items for Systematic Reviews and Meta-Analyses (PRISMA) [20] flow diagram describing the steps for including studies in the review. ICS: inhaled corticosteroids; LABA: long-acting $\beta_{2}$-agonist. 
TABLE 1 Characteristics of the 23 studies using objective measures to assess adherence

\begin{tabular}{|c|c|c|c|c|c|c|}
\hline First author [ref.] & Design & $\begin{array}{l}\text { Age } \\
\text { years }\end{array}$ & $\begin{array}{c}\text { Participants } \\
\text { n }\end{array}$ & $\begin{array}{l}\text { Adherence } \\
\text { measure }\end{array}$ & $\begin{array}{l}\text { Adherence } \\
\text { cut-off }\end{array}$ & $\begin{array}{c}\text { Exacerbation } \\
\text { measure }\end{array}$ \\
\hline \multicolumn{7}{|c|}{$\begin{array}{l}\text { Refill data (MPR and } \\
\text { prescriptions) } \\
\text { Children }\end{array}$} \\
\hline Rust [21] & Cohort & $5-12$ & 43166 & $\begin{array}{l}\text { ICS PPDC } \\
90 \text { days; } \\
\text { ratio controller } \\
\text { to total drugs }\end{array}$ & $\begin{array}{l}\text { PPDC: } \geqslant 50 \% \\
\text { or }<50 \% \text {; } \\
\text { ratio: } \geqslant 0.5 \\
\quad \text { or }<0.5\end{array}$ & ED/hosp \\
\hline Camargo [22] & Cohort & $0-8$ & 10976 & MPR ICS & $\begin{array}{l}\text { Dichotomised } \\
\text { at median } \\
\text { MPR: } 0.08\end{array}$ & $\begin{array}{l}\text { Combined: ED/ } \\
\text { hosp visit }\end{array}$ \\
\hline BUKSTEIN [23] & Cohort & $0-4$ & 11407 & $\begin{array}{l}\text { Number of ICS } \\
\text { prescriptions }\end{array}$ & $\begin{array}{c}\geqslant 2 \text { versus } 1 \\
\text { prescription per } \\
9 \text { months before } \\
\text { index date }\end{array}$ & $\begin{array}{c}\text { Combined: ED/ } \\
\text { hosp }\end{array}$ \\
\hline BUKSTEIN [24] & $\begin{array}{l}\text { Randomised } \\
\text { cohort }\end{array}$ & $6-15$ & 104 & Filled prescriptions & & $\begin{array}{l}\text { ED/hosp/office } \\
\text { visit/OCS }\end{array}$ \\
\hline ELKoUt [25] & Cohort & Children & 3172 & MPR ICS & $\begin{array}{c}\text { Adequate MPR } 80- \\
120 \%\end{array}$ & OCS \\
\hline HERNDON [26] & Cohort & $2-18$ & 10878 & MPR ICS & $\begin{array}{c}3 \text { MPR categories: } \\
0-19 \%, 20-49 \% \\
>50 \%\end{array}$ & ED/hosp \\
\hline \multicolumn{7}{|l|}{ Adults } \\
\hline PRICE [27] & $\begin{array}{l}\text { Retrospective } \\
\text { matched } \\
\text { cohort }\end{array}$ & $12-80$ & 30939 & $\begin{array}{l}\text { Beclomethasone } \\
\text { MPR; } \\
\text { ratio controller } \\
\text { to total }\end{array}$ & $\begin{array}{c}4 \text { MPR categories: } \\
<50 \%, 50-70 \% \\
70-99 \%,>100 \% \\
\text { ratio: }<0.5 \text { or } \geqslant 0.5\end{array}$ & ED/hosp; OCS \\
\hline Williams [28] & $\begin{array}{c}\text { Prospective } \\
\text { asthma cohort }\end{array}$ & $12-56$ & 298 & Moving CMA ICS & $\begin{array}{c}\text { Per } 25 \% \text { increase } \\
\text { MPR }\end{array}$ & $\begin{array}{c}\text { Combined and ED/ } \\
\text { hosp/OCS }\end{array}$ \\
\hline
\end{tabular}

Estimates

Quality

$$
\begin{gathered}
\text { Exacerbation nonadherent versus } \\
\text { adherent ratio: } \\
\text { ORadjED } 1.21 \text { (95\% Cl 1.14-1.27); } \\
\text { ORadjhosp 1.70 (95\% Cl 1.45-1.98) }
\end{gathered}
$$$$
\text { ORadjED } 1.21 \text { (95\% Cl 1.14-1.27): }
$$

Exacerbation adherent versus nonadherent;$$
\begin{aligned}
& \text { budesonide HRadj } 0.32 \text { (9 } \\
& \text { Cl 0.19-0.68); }
\end{aligned}
$$

non-nebulised ICS HRadj 0.25 (95\% $\mathrm{Cl} 0.13-0.47)$

Exacerbations: adherent versus less adherent;

all controller therapy: OR $0.80(95 \% \mathrm{Cl} 0.59-1.10)$ :

$$
\text { ICS only: OR } 0.60(95 \% \mathrm{Cl} 0.37-0.99)
$$

Adherent $(\geqslant 6)$ versus nonadherent ( $\leqslant 5$ fills); OCS: $26 \%$ adherent versus $44 \%$ nonadherent; montelukast users more adherent than

fluticasone users ( $p=0.0003$ ); no significant difference in hosp/ED/office

visits between montelukast/fluticasone groups

Adequate MPR (80-120\%) was associated

with higher risk of being prescribed OCS (NS);

ICS only: ORadj 1.02 (95\% Cl 1.00-1.04);

LABA/ICS: ORadj 1.12 (95\% Cl 0.58-2.11):

LABA+ICS: ORadj $1.43(95 \% \mathrm{Cl} 0.75-2.71)$

Higher adherence, less ED $(p=0.01)$

$M P R \geqslant 0.50$ versus $\leqslant 0.19$ :

ED: ORadj 0.56 (95\% Cl 0.43-0.72);

hosp: ORadj 0.96 [95\% Cl 0.67-1.36)

Higher exacerbation rates by better adherence to ICS

$25 \%$ increased adherence:

combined outcome: HRadj $0.89195 \% \mathrm{Cl}$

$0.81-0.97), p=0.009$

OCS: HRadj $0.90(95 \% \mathrm{Cl} 0.80-1.0), p=0.043$;

ED: HRadj 0.87 (95\% Cl 0.73-1.03), $\mathrm{p}=0.114$;

hosp: HRadj 0.99 (95\% Cl 0.65-1.51), $\mathrm{p}=0.971$.

High $(76-100 \%$ MPR) versus low $(0-25 \%$ MPR):

OR $0.58(0.39-0.87)$ 


\begin{tabular}{|c|c|c|c|c|c|c|c|c|}
\hline First author [ref.] & Design & $\begin{array}{l}\text { Age } \\
\text { years }\end{array}$ & $\begin{array}{l}\text { Participants } \\
\quad \mathrm{n}\end{array}$ & $\begin{array}{l}\text { Adherence } \\
\text { measure }\end{array}$ & $\begin{array}{l}\text { Adherence } \\
\text { cut-off }\end{array}$ & $\begin{array}{l}\text { Exacerbation } \\
\text { measure }\end{array}$ & Estimates & $\begin{array}{l}\text { Quality } \\
\text { score }\end{array}$ \\
\hline WiLLIAMs [29] & Cohort & $18-50$ & 405 & CMA; CMG; for ICS & $\begin{array}{l}\text { Per } 25 \% \text { increase } \\
\text { in } \mathrm{CMA} / \mathrm{CMG}\end{array}$ & $\begin{array}{l}\text { Outpatient/ED/ } \\
\text { hosp/OCS }\end{array}$ & $\begin{array}{c}\text { Per } 25 \% \text { increase gap: } \\
\text { ED: RRadj } 1.25(95 \% \mathrm{Cl} 0.84-1.85) ; \\
\text { OCS: RRadj } 1.26(95 \% \mathrm{Cl} 0.95-1.67) ; \\
\text { hosp: RRadj } 2.01(95 \% \mathrm{Cl} 1.06-3.79) . \\
\text { Per } 25 \% \text { increase CMA: } \\
\text { OCS: RR 0.75 (95\% Cl 0.58-0.97). } \\
\text { Correlation adherence CMA: } \\
\text { ED: R -0.159, OCS: R -0.179, hosp: }-0.130 \text { (NS) }\end{array}$ & 8 \\
\hline BALKRISHNAN [30] & Case-control & $\begin{array}{l}\text { Older } \\
\text { adults }\end{array}$ & 751 & $\begin{array}{l}\text { Filled prescription } \\
\text { ICS }\end{array}$ & $\begin{array}{l}0,1 \text { or } 2 \text { refills in } 2 \\
\text { months before } \\
\text { event }\end{array}$ & $\begin{array}{l}\text { Combined: ED/ } \\
\text { hosp }\end{array}$ & $\begin{array}{l}\text { Exacerbation (referent=nonadherent=0 refills) } \\
\text { ORadj good ( } 2 \text { refills ICS): } 0.62(95 \% \mathrm{Cl} 0.42-0.90) \text {; } \\
\text { ORadj partial (1 refill ICS): } 0.75(95 \% \mathrm{Cl} 0.57-0.96)\end{array}$ & 8 \\
\hline MATTKE [31] & Cohort & $0-65$ & 12476 & MPR ICS & $\begin{array}{l}\text { MPR quarters: } \\
\text { highest versus } \\
\text { lowest }\end{array}$ & $\begin{array}{l}\text { Combined: ED/ } \\
\text { hosp/office }\end{array}$ & $\begin{array}{c}\text { Lowest versus highest adherence quarters; } \\
\text { incidence of ED and hosp: not } \\
\text { significantly different }\end{array}$ & 8 \\
\hline DeLEA [32] & Cohort & $\geqslant 12$ & 12907 & MPR FSC & $\begin{array}{l}\text { MPR quartiles per } \\
3 \text { months of } \\
\text { follow-up }\end{array}$ & ED/hosp or OCS & $\begin{array}{l}\text { Per } 25 \% \text { increase mean adherence: } \\
\text { ED/hosp: ORadj } 0.90 \text { (95\% Cl 0.89-0.92); } \\
\text { OCS: ORadj } 0.97(95 \% \mathrm{Cl} 0.94-0.996)\end{array}$ & 8 \\
\hline Stern [33] & Cohort & $6-99$ & 97743 & MPR all controllers & & Combined ED/hosp & $\begin{array}{l}\text { 75th percentile MPR cut-off versus less adherent; } \\
\text { exacerbation ORadj } 0.862(95 \% \mathrm{Cl} 0.827-0.898)\end{array}$ & 8 \\
\hline McMaHON [34] & Cohort & $12-45$ & 4535 & $\begin{array}{c}\text { Days with ICS per } 90 \\
\text { days }\end{array}$ & $\begin{array}{l}0 \text { days ICS; } 1-89 \\
\text { days; } 90 \text { days }\end{array}$ & $\begin{array}{l}\text { Combined: } \\
\text { hosp+OCS and } \\
\text { hosp only }\end{array}$ & $\begin{array}{c}\text { Combined exacerbation: } \\
0 \text { days adherent: } 0 \text { Radj } 0.77(95 \% \\
\text { Cl } 0.44-1.35) ; \\
\text { 1-89 days adherent: ORadj } 1.02(95 \% \mathrm{Cl} 0.60-1.73) . \\
\text { Hosp: } 0 \text { days adherent: ORadj } 1.12(95 \% \mathrm{Cl} 0.36-3.47) ; \\
\text { 1-89 days adherent: ORadj } 0.91(95 \% \\
\mathrm{Cl} 0.31-2.72 \text { ) }\end{array}$ & 7 \\
\hline SмIтн [35] & Cohort & $5-62$ & 3013 & MPR all controllers & $\begin{array}{l}\text { MPR: } 0-50 \% \\
50-80 \% \text { or }>80 \%\end{array}$ & ED/hosp & $\begin{array}{l}\text { Risk of admission (ED/hosp) nonadherent versus: } \\
50-80 \% \text { adherent: OR } 1.59 \text { ( } 95 \% \mathrm{Cl} 0.86-2.96) \text {; } \\
>80 \% \text { highly adherent: OR } 2.11(95 \% \mathrm{Cl} 1.09-4.12)\end{array}$ & 7 \\
\hline HYLAND [36] & Cohort & Adults & 166 & $\begin{array}{l}\text { Prescription and } \\
\quad \text { records }\end{array}$ & $\begin{array}{l}>75 \% \\
\text { recommended } \\
\text { prescriptions }\end{array}$ & $\begin{array}{l}\text { Combined: } \\
\text { GP visit/ED/hosp }\end{array}$ & $\begin{array}{l}\text { Spearman correlation between asthma } \\
\text { exacerbations and adherence } 0.21(p=0.007)\end{array}$ & 6 \\
\hline Osman [37] & Prospective cohort & Adults & 754 & $\begin{array}{l}\text { Requested } \\
\text { prescription }\end{array}$ & $\begin{array}{l}\text { Among patients } \\
\text { with <7 SABA: } \\
\leqslant 4 \text { ICS versus } \\
5-7 \text { ICS versus } \\
\geqslant 8 \text { ICS }\end{array}$ & Hosp/OCS & $\begin{array}{l}<7 \text { SABA and } \leqslant 4 \text { ICS versus } 5-7 \text { ICS } \\
\text { and } \geqslant 8 \text { ICS: used few OCS }(p=0.06) ; \\
\text { more hospital admissions }(p<0.05)\end{array}$ & 5 \\
\hline \multicolumn{9}{|c|}{$\begin{array}{l}\text { Electronic monitoring } \\
\text { device (children only) }\end{array}$} \\
\hline RoHAN [38] & Prospective cohort & $5-17$ & 92 & $\begin{array}{l}\text { Electronic } \\
\text { monitoring device } \\
\text { ICS }\end{array}$ & $\begin{array}{l}\text { Daily ICS use } \\
\text { averaged over } \\
\text { 5-day intervals }\end{array}$ & $\begin{array}{l}\text { Healthcare visits } \\
\text { (ED/hosp/visit } \\
\text { specialist) }\end{array}$ & $\begin{array}{c}\text { Growth curve modelling: average } \\
\text { healthcare-related visits per year } \\
\text { low adherent ( } 1 \text { sD below mean): } 0.76 ; \\
\text { moderate adherent: } 0.70 ; \\
\text { good adherent (1 SD above mean): } 0.65\end{array}$ & 6 \\
\hline McNalLy [39] & Cohort & $5-17$ & $\begin{array}{l}63 \text { ICS+LTRA } \\
\text { users }\end{array}$ & $\begin{array}{l}\text { Electronic } \\
\text { monitoring device } \\
\text { ICS adherence rate } \\
\text { (mean \% prescribed) }\end{array}$ & $\begin{array}{l}\text { Highest quartile } \\
\text { (mean 0.62) versus } \\
\text { lowest quartile } \\
\text { (mean } 0.20 \text { ) }\end{array}$ & $\begin{array}{l}\text { Healthcare } \\
\text { utilisation (hosp/ } \\
\text { ED/clinic visit) }\end{array}$ & $\begin{array}{c}\text { Decline in fluticasone adherence was related to increased } \\
\text { healthcare utilisation: } p<0.05 ; \\
\text { rate of change in healthcare utilisation } \\
\text { related to fluticasone low versus high } \\
\text { adherence: } R=-0.11, p=0.39 \text { (NS) }\end{array}$ & 6 \\
\hline
\end{tabular}




\begin{tabular}{|c|c|c|c|c|c|c|c|c|}
\hline First author [ref.] & Design & $\begin{array}{l}\text { Age } \\
\text { years }\end{array}$ & $\begin{array}{l}\text { Participants } \\
\text { n }\end{array}$ & $\begin{array}{l}\text { Adherence } \\
\text { measure }\end{array}$ & $\begin{array}{l}\text { Adherence } \\
\text { cut-off }\end{array}$ & $\begin{array}{l}\text { Exacerbation } \\
\text { measure }\end{array}$ & Estimates & $\begin{array}{l}\text { Quality } \\
\text { score }\end{array}$ \\
\hline MILGROM [9] & $\begin{array}{l}\text { Cross- } \\
\text { sectional } \\
\text { (4 visits) }\end{array}$ & $8-12$ & 24 & $\begin{array}{c}\text { Electronic } \\
\text { monitoring } \\
\text { device ICS and } \\
\beta_{2} \text {-agonist }\end{array}$ & NA & OCS & $\begin{array}{l}\text { Mean adherence } 13.7 \% \text { in cases versus } \\
68.2 \% \text { in patients without OCS } \\
\text { ( } p=0.008)\end{array}$ & 3 \\
\hline \multicolumn{9}{|l|}{$\begin{array}{l}\text { Weighing/counting } \\
\text { Children }\end{array}$} \\
\hline KRISHNAN [40] & $\begin{array}{l}\text { Randomised } \\
\text { controlled } \\
\text { trial }\end{array}$ & $5-12$ & 140 & $\begin{array}{l}\text { Weighing/counting } \\
\text { budesonide/placebo }\end{array}$ & NA & $\mathrm{ED} / \mathrm{OCS}$ & $\begin{array}{l}\text { Treatment group, adherence } 4 \text { years, } \\
\text { interaction: ED visits (yes versus no), OR } p=0.58 \text {; } \\
\text { OCS (number of courses } \\
\text { per } 100 \text { person-years) } p=0.56\end{array}$ & 9 \\
\hline LASMAR [41] & Prospective cohort & $3-12$ & 122 & $\begin{array}{l}\text { Weighing/counting } \\
\text { beclomethasone }\end{array}$ & NA & $\begin{array}{l}\text { Combined: asthma } \\
\text { deterioration, OCS, ED, } \\
\text { hosp }\end{array}$ & $\begin{array}{l}\text { Adherence level } 70.9 \% \text { in group of } \\
\text { patients without exacerbation versus } \\
44 \% \text { in group of patients with exacerbations } \\
\qquad(p=0.004)\end{array}$ & 5 \\
\hline \multicolumn{9}{|c|}{ 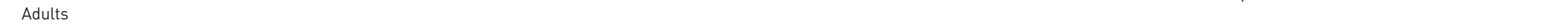 } \\
\hline Santos [42] & Prospective cohort & $>18$ & 160 & $\begin{array}{l}\text { Weighing/counting } \\
\text { ICS }\end{array}$ & $\begin{array}{l}\text { Cut-off point: } 80 \% \\
\text { of prescribed dose } \\
\text { administered }\end{array}$ & Exacerbation/ED & $\begin{array}{l}\text { Adherent versus nonadherent: } \\
\text { exacerbation: } 45.5 \% \text { versus } 50 \% \text { (Ns); } \\
\text { mean } \pm \text { SD ED visits: } 0.9 \pm 1.9 \text { versus } \\
1.4 \pm 2.6(p=0.2)\end{array}$ & 3 \\
\hline $\begin{array}{l}\text { MPR: medication } \\
\text { ratio; HRadj: adju } \\
\text { gaps; RRadj: adju } \\
\text { applicable. }\end{array}$ & $\begin{array}{l}\text { ssession rate; IC } \\
d \text { hazard ratio; } 0 \\
\text { d relative rate; } F\end{array}$ & inhale & $\begin{array}{l}\text { corticoster } \\
\text { prticosteroid } \\
\text { sone/salme }\end{array}$ & $\begin{array}{l}\text { ids; PPDC: proporti } \\
\text { 5; NS: nonsignificant } \\
\text { erol combination; } G\end{array}$ & $\begin{array}{l}\text { on of prescribed d } \\
\text { : LABA: long-actin } \\
\text { P: general practiti }\end{array}$ & $\begin{array}{l}\text { lays covered; ED: eme } \\
\text { g } \beta_{2} \text {-agonist; CMA: } c \\
\text { oner; SABA: short-ac }\end{array}$ & $\begin{array}{l}\text { department; hosp: hospitalisation; ORadj: } \\
\text { us measure of availability; CMG: continuo } \\
\text {-agonist; LTRA: leukotriene receptor anta }\end{array}$ & $\begin{array}{l}\text { ed odds } \\
\text { asure of } \\
\text { NA: not }\end{array}$ \\
\hline
\end{tabular}


assess adherence. Of the remaining studies, three used canister weighing/counting and three used electronic monitoring via a specific device.

\section{Measures of severe asthma exacerbations}

Of the 23 studies using objective measures to assess adherence, five defined exacerbation as emergency department visit and/or hospitalisation and/or oral corticosteroids (OCS), and 16 defined exacerbation as two out of three of these criteria. Five studies defined the need for OCS as a separate outcome. Only one study had need for OCS as the only outcome.

\section{Asthma diagnosis}

In four studies, the asthma diagnosis was based on physician diagnosis. In the remaining studies, diagnoses were based on disease codes (International Classification of Diseases, 9th revision (ICD-9)) $(n=9)$, disease codes and prescriptions $(n=2)$, asthma controller therapy prescriptions $(n=3)$, guidelines $(n=4)$, or study-specific asthma definitions $(n=1)$.

\section{Adherence and exacerbations}

Due to differences in design, exposure, outcome, cut-off values, assessments and definitions, studies differed substantially in effect magnitudes and this precluded a formal meta-analysis with pooling of results. Therefore, we report the findings separately for each type of objective adherence measure. The order of reporting within each category is based on quality score, indicated with the letter q. We reported results separately for children and adults. Details of the 23 studies included in this review are summarised in table 1 . The overall conclusions remained similar when papers with low quality $(q<6)$ were excluded.

An overview of the range of risk estimates, for those studies that reported risk estimates for the association between adherence and asthma exacerbation, is shown in figure 2 (for children) and figure 3 (for adults).

\section{Objective adherence measurements}

To assess overall treatment adherence, 17 studies used refill data; of these, 11 used MPR measurements $[21,22,25-29,31-33,35]$ and six used number of prescription refills [23, 24, 30, 34, 36, 37]. The remaining studies used either electronic monitoring devices $(n=3)[9,38,39]$ or weighted canisters $(n=3)$ [40-42].

The overall treatment adherence was low in paediatric and adult studies. In children, adherence measured as average MPR was only $20-33.9 \%$ for ICS $[26,38]$. The number of prescription fillings over the course of 1 year ranged from 4.7 to 5.5 times for fluticasone $[24,37]$. In adults, MPR for ICS ranged between $15 \%[26,31]$ and $54 \%[32]$.

WiLliams et al. [28] demonstrated, with a moving 6-month average ICS adherence for each day of follow-up, that adherence to ICS medications began to increase just before the first asthma exacerbation, and continued to increase after the event.

Association between exacerbation and objective adherence in paediatric studies (refill data) Four out of six paediatric cohort studies using refill data reported that the risk of asthma exacerbation was 21-68\% lower for children who were more adherent to their asthma controller medication compared with

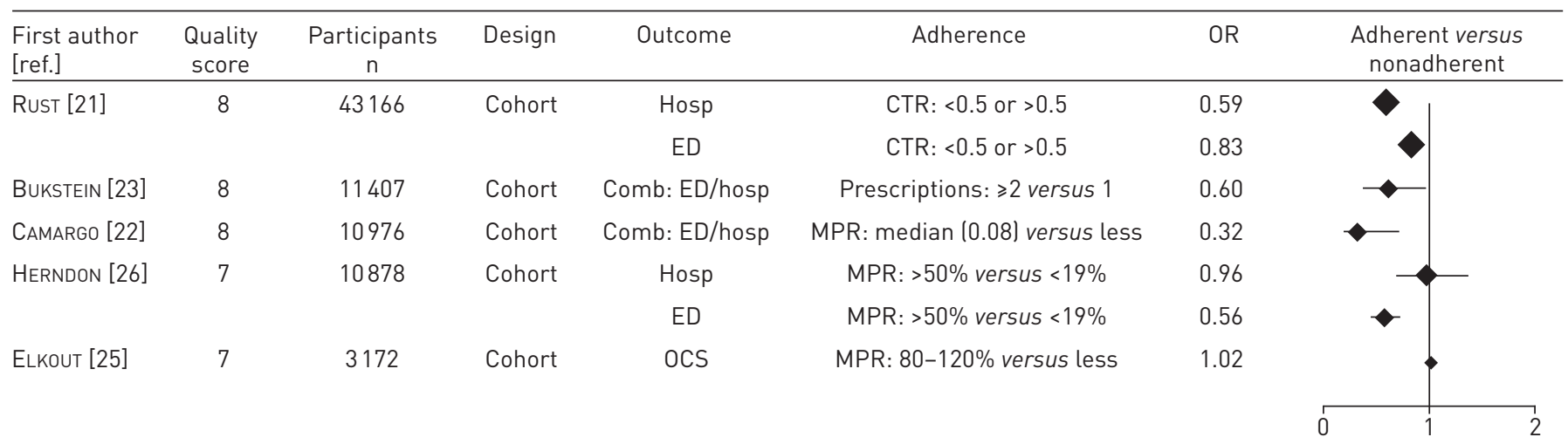




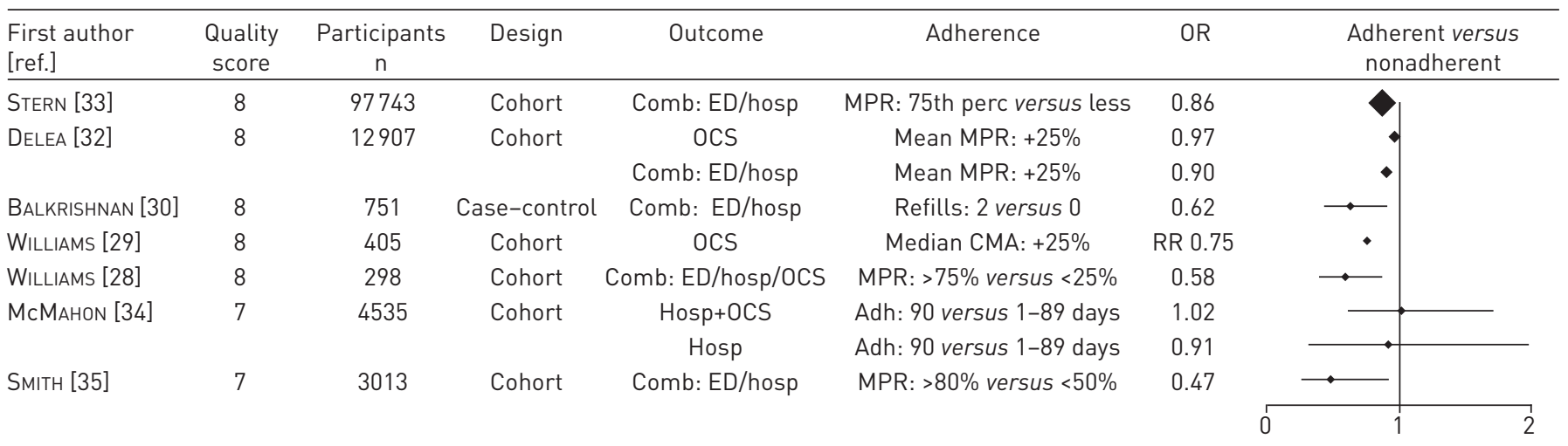

FIGURE 3 Overview of adult studies publishing odds ratios (OR) or relative rate (RR), sorted by quality score, number of participants and year published. Comb: combined; ED: emergency department visit for asthma; hosp: hospitalisation for asthma; MPR: medication possession rate; perc: percentile; OCS: oral corticosteroids; CMA: continuous measure of availability; Adh: adherence.

those who were less adherent [21-23, 26]. Rust et al. [21] $(\mathrm{q}=8)$ observed this protective effect only if adherence was measured as controller-to-total ratio. In contrast, if adherence was measured as proportion of prescribed days covered (PPDC), lower PPDC was associated with lower emergency department/ hospitalisation exacerbations.

A previous study in 2003 by BuKstein et al. [24] ( $q=7$ ) found no significant differences between fluticasone and montelukast users (who were more adherent) in the frequency of asthma attacks requiring hospital admissions, acute office visits or emergency department visits, but found a significant difference in the amount of OCS, which was higher for fluticasone than for montelukast.

Finally, ElKout et al. [25] ( $q=7$ ) reported that the risk of being prescribed OCS in the year after first prescription of controller therapy was $2-43 \%$ higher in children with adequate MPR than those with inadequate MPR, although this difference was not statistically significant.

Association between exacerbation and objective adherence in adult studies (refill data)

Six out of 11 studies reporting on refills in adults showed that better adherence was associated with a reduced risk of severe asthma exacerbations. The four adult studies of good quality ( $q=8)$, reporting MPRs $[28,29,32,33]$ concluded that $25 \%$ increased adherence was associated with approximately $10 \%$ reduction in severe exacerbations (adjusted hazard ratio 0.89 [28], relative rate 0.75 [29], adjusted odds ratios 0.90 [32] and 0.86 33). Similar associations were reported by MatтKe et al. [31] ( $q=8)$ for leukotriene receptor antagonist users but not for ICS users. Interestingly, BALKRISHNAN et al. [30] ( $q=8$ ) showed, in ICS users, a $40 \%$ decreased risk of an emergency department visit/hospitalisation in adherent versus nonadherent elderly patients with chronic pulmonary illnesses, including asthma. In contrast, five cohort studies (q range 5-9) reported that the risk of OCS use and/or hospitalisation and/or emergency department visits increased with better adherence [27, 34-37].

\section{Association between exacerbation and other measures of objective adherence}

Three studies, two paediatric ( $\mathrm{q}=5$ and $\mathrm{q}=9$ ) and one adult $(\mathrm{q}=3$ ), measured adherence by counting/ weighing pills and canisters at the pharmacy or outpatient clinic [40-42]. Only one of these reported that adherence was associated with a reduced risk of asthma exacerbation [41].

Three paediatric studies (q range 3-6) measured adherence through electronic device monitoring, and all of these reported an association between low adherence and increased risk of severe asthma exacerbations, hospitalisations/emergency department visits [38, 39] or OCS [9].

\section{Association between OCS courses and objective adherence}

Seven studies, three paediatric and four adult, assessed adherence in relation to the need for an OCS course as a separate outcome. In four of these $(\mathrm{q}=7$ or $\mathrm{q}=8$ ), the need for OCS courses was inversely related to the controller adherence rate, both in children and adults [24, 28, 29, 32]. In the remaining three studies (q range 5-9), this association was not significant in one paediatric study [40], and in one paediatric and one adult study an opposite, nonsignificant trend was observed $[25,37]$. 


\section{Discussion}

To our knowledge, this is the first systematic review on the association between treatment adherence and severe asthma exacerbations. In the identified articles, we observed that low adherence was common. Despite heterogeneity among studies in terms of definitions of adherence and asthma exacerbations, the majority of the high-quality studies consistently reported an association between low adherence and higher risk of severe asthma exacerbations, both in adults and children.

We identified important differences and limitations in the included studies. Notably, the majority of studies were classified as moderate to low quality, largely because of flaws in the study methods, e.g. small sample sizes, which may compromise power, and cross-sectional designs that are prone to bias. When excluding studies of poor or moderate quality $(\mathrm{q}<6)$ or excluding studies that used a design other than cohort design, the overall conclusion remained the same, namely that good adherence was associated with fewer severe asthma exacerbations.

Another limitation was that the included studies varied widely with regard to the definition of adherence. There is no standardised method to measure adherence, and each measure has its own strengths and limitations [11,44,45]. Objective measures are considered to be more reliable and accurate than indirect measures [11]. Electronic device monitoring is usually considered the gold standard because of a detailed assessment of adherence patterns. Although it has the benefit of being able to identify and exclude "dumping" (deliberate emptying of inhaler before study visits to conceal nonadherence) from analyses, unfortunately such monitoring is expensive and often prone to device failure [11, 35]. The most cost-effective method to assess adherence is by self-report. However, the reliability of this method is questionable [46], as it is well known that patients over-report their adherence [9, 11, 47], even in a clinical trial setting [40]. Hence, self-report was not included in this study.

The majority of studies calculated the MPR, which is a common way to measure medication adherence from claims or pharmacy data that has been found to be useful and reasonably accurate [48, 49]. MPR data have the advantage of being easily accessible and inexpensive [45]. The drawback of these databases is that details about devices and day-to-day patterns of adherence are not always recorded [11, 45].

Another method used to measure adherence was the number of prescriptions, but this is highly influenced by the level of asthma control. Indeed, patients appear to self-titrate their medication, showing greater adherence to therapy when they have a worse level of control [27]. Additionally, the number of prescriptions is also influenced by the quality and reliability of physicians prescribing. The best method to tease this out is by using a ratio of long-term controller to total asthma medications, in which the denominator and numerator increase with increasing severity, but the numerator is more specifically reflective of adherence to therapy [21].

Even if studies used a common methodology to assess adherence, a wide variety of cut-off values to define "adherent patients" was observed. This arbitrary selection of adherence cut-off points is of considerable concern. Traditionally, medication adherence is dichotomised using a cut-off value (e.g. $80 \%)$, which is derived from studies in other chronic diseases such as HIV and hypertension [50] but does not necessarily hold for asthma.

It was quite remarkable that most studies did not attempt to measure whether and how medication was actually taken. Inhaler competence, the skill to inhale correctly, is particularly relevant for asthma medication, as inhaling of drugs requires considerable skill and practice [51]. Even if medication is taken daily, deposition in the lungs will be low with incorrect inhalation technique [52]. Newer devices such as breath-actuated inhalers and smart nebulisers, which impose breathing patterns and record lung deposition, may partly overcome this problem, but still require a high level of cooperation. Smart nebulisers are expensive and the experience in children with asthma is limited [53]. Nevertheless, all of these methods can overestimate adherence and there remains a number of complex issues affecting optimal medication use in children with asthma, e.g. a lack of parental knowledge about asthma medications, parental beliefs and fears, and the child's self-image [54].

We noted heterogeneity in outcome definitions, despite international guidelines on the definitions and assessment of severe asthma exacerbations [55]. This might partly be explained by the data being used, for example pharmacy data do not always have detailed information on disease codes and/or symptoms and diagnosis. As asthma is a difficult diagnosis, especially in children, risk of asthma misclassification is high if based on prescription data only.

In a pragmatic trial, patients are randomised within a real-life setting that combines the sound methodology of randomised controlled trials with daily practice, enlarging the external validity of study results. This external validity is crucial for studies investigating treatment adherence and associated factors [56]. All methods have their sets of benefits and limitations [57]. It has been suggested that the 
best design would probably be a combination of observational studies, pragmatic trials and randomised controlled trials, as all have advantages and drawbacks and they are not designed to answer the same questions [58].

Some of the studies were at risk of bias. There are limitations arising from the observational nature of the included studies. One important bias is the "healthy adherer effect", where healthier people are predisposed to follow medical advice, because they are more concerned about their health. Other biases in this type of research are recall bias and observer/investigator bias when using questionnaires [59]. Furthermore, misclassification of adherence caused by dumping or over-reporting might have contributed to an underestimation of the role of adherence in mediating the observed treatment effects.

Another limitation was that some studies did not adjust for potential confounders. "Asthma severity" is an important confounder that is difficult to control for, as details on asthma severity are often lacking in database studies. Other potential confounders are environmental factors, such as pollution or smoking habits of the parents.

Although the majority of papers of good quality indicated that higher levels of adherence were associated with a reduced risk of severe asthma exacerbations, this was not confirmed in all studies. Some studies even reported an inverse association between treatment adherence and risk of severe asthma exacerbations. This might be explained by the fact that the number of prescriptions was used as a proxy for adherence $[35,37]$, or because unusual cut-off values for adherence were used [34]. Furthermore, there are general potential explanations for this inverse association. Treatment need is higher in patients with poorly controlled asthma, who are by definition at risk of asthma exacerbation. This higher need for treatment will result in increased prescription of asthma controller therapies, with a consequently higher MPR [37, 46]. Another explanation is self-titrating of medications: patients show greater adherence to therapy with worse levels of control, thus positively influencing the MPR [27]. A third explanation could be the heterogeneity among asthma patients in treatment response: some patients reduce their prescribed controller medication without negative consequences [37], whereas other patients continue to have poor outcomes despite good adherence [60]. Additionally, there is no known dose of medication or duration of treatment. Therefore, the "low" adherence shown by some people may be adequate for them most of the time.

The main strengths of this review are the comprehensiveness of the searches and our standardised approach to study selection, data extraction and quality assessment. However, our review has some limitations that need to be considered. Only published data was included, and the relatively small number of relevant publications suitable for this review warrants caution given the possibility of publication bias. Publication bias is a type of error that may affect the results of a meta-analysis because studies with statistically significant positive findings are more likely to be published than studies with negative results [61]. However, given the heterogeneity, inclusion of additional studies would have been unlikely to undermine our overall conclusion.

Overall, this review highlights the importance of adherence to prevent severe asthma exacerbations. This is in agreement with recent predictive models, which observed that improvements in medication adherence could lead to significant improvements in asthma outcomes [62]. It is clear that better studies about adherence to treatments are needed, and that more homogeneous outcomes and study design would be useful in order to reach conclusions. For clinicians, it seems evident that efforts directed at improving, evaluating and measuring adherence should be a routine component of asthma care [63]. The recognition of nonadherence is an important first step towards optimal asthma control.

\section{Conclusion}

Despite the limitations, in this review the majority of the papers of good quality indicated that higher levels of adherence were associated with a reduced risk of severe asthma exacerbations. Adherence to asthma controller therapy was generally very low. To further elucidate the association between adherence and risk of asthma exacerbations, there is a need for new, well designed real-life prospective studies, using consistent standardised measures for both treatment adherence (preferably electronic monitoring) and asthma exacerbations. Future research should also include inhaler competence, as it is an important confounder in the association of interest, and essential to disentangle the association between adherence and treatment outcomes.

\section{References}

1 Pearce N, Aït-Khaled N, Beasley R, et al. Worldwide trends in the prevalence of asthma symptoms: phase III of the International Study of Asthma and Allergies in Childhood (ISAAC). Thorax 2007; 62: 758-766.

2 To T, Stanojevic S, Moores G, et al. Global asthma prevalence in adults: findings from the cross-sectional world health survey. BMC Public Health 2012; 12: 204. 
Bender BG, Rand C. Medication non-adherence and asthma treatment cost. Curr Opin Allergy Clin Immunol 2004; 4: 191-195.

4 Bateman ED, Hurd SS, Barnes PJ, et al. Global strategy for asthma management and prevention: GINA executive summary. Eur Respir J 2008; 31: 143-178.

5 Choi TN, Westermann H, Sayles W, et al. Beliefs about asthma medications: patients perceive both benefits and drawbacks. J Asthma 2008; 45: 409-414.

6 Harrison B, Stephenson P, Mohan G, et al. An ongoing Confidential Enquiry into asthma deaths in the Eastern Region of the UK, 2001-2003. Prim Care Respir J 2005; 14: 303-313.

7 Horne R. Compliance, adherence, and concordance: implications for asthma treatment. Chest 2006; 130: Suppl. 1, 65S-72S.

8 Côté I, Farris K, Feeny D. Is adherence to drug treatment correlated with health-related quality of life? Qual Life Res 2003; 12: 621-633.

9 Milgrom H, Bender B, Ackerson L, et al. Noncompliance and treatment failure in children with asthma. J Allergy Clin Immunol 1996; 98: 1051-1057.

10 Bender BG, Bender SE. Patient-identified barriers to asthma treatment adherence: responses to interviews, focus groups, and questionnaires. Immunol Allergy Clin North Am 2005; 25: 107-130.

11 Rand CS, Wise RA. Measuring adherence to asthma medication regimens. Am J Respir Crit Care Med 1994; 149: S69-S76.

12 Williams LK, Joseph CL, Peterson EL, et al. Patients with asthma who do not fill their inhaled corticosteroids: a study of primary nonadherence. J Allergy Clin Immunol 2007; 120: 1153-1159.

13 Chan PW, DeBruyne JA. Parental concern towards the use of inhaled therapy in children with chronic asthma. Pediatr Int 2000; 42: 547-551.

14 Allen DB. Inhaled steroids for children: effects on growth, bone, and adrenal function. Endocrinol Metab Clin North Am 2005; 34: 555-564.

15 Peterson AM, Nau DP, Cramer JA, et al. A checklist for medication compliance and persistence studies using retrospective databases. Value Health 2007; 10: 3-12.

16 Reddel HK, Taylor DR, Bateman ED, et al. An official American Thoracic Society/European Respiratory Society statement: asthma control and exacerbations: standardizing endpoints for clinical asthma trials and clinical practice. Am J Respir Crit Care Med 2009; 180: 59-99.

17 Taylor DR, Bateman ED, Boulet LP, et al. A new perspective on concepts of asthma severity and control. Eur Respir J 2008; 32: 545-554.

18 Goodman M, Hays S. Asthma and swimming: a meta-analysis. J Asthma 2008; 45: 639-647.

19 Wells GA, Shea B, O'Connell D, et al. The Newcastle-Ottawa Scale (NOS) for assessing the quality of nonrandomised studies in meta-analyses. www.ohri.ca/programs/clinical_epidemiology/oxford.asp

20 Moher D, Liberati A, Tetzlaff J, et al. Preferred reporting items for systematic reviews and meta-analyses: the PRISMA statement. PLoS Med 2009; 6: e1000097.

21 Rust G, Zhang S, Reynolds J. Inhaled corticosteroid adherence and emergency department utilization among Medicaid-enrolled children with asthma. J Asthma 2013; 50: 769-775.

22 Camargo CA Jr, Ramachandran S, Ryskina KL, et al. Association between common asthma therapies and recurrent asthma exacerbations in children enrolled in a state Medicaid plan. Am J Health Syst Pharm 2007; 64: 1054-1061.

23 Bukstein DA, Murphy KR, Katz LM, et al. Outcomes among a young population of pediatric asthma patients using controller therapies: results from a retrospective database analysis. Pediatr Asthma Allergy Immunol 2007; 20: 211-222.

24 Bukstein DA, Luskin AT, Bernstein A. "Real-world" effectiveness of daily controller medicine in children with mild persistent asthma. Ann Allergy Asthma Immunol 2003; 90: 543-549.

25 Elkout H, Helms PJ, Simpson CR, et al. Adequate levels of adherence with controller medication is associated with increased use of rescue medication in asthmatic children. PLoS One 2012; 7: e39130.

26 Herndon JB, Mattke S, Evans Cuellar A, et al. Anti-inflammatory medication adherence, healthcare utilization and expenditures among Medicaid and children's health insurance program enrollees with asthma. Pharmacoeconomics 2012; 30: 397-412.

27 Price D, Thomas M, Haughney J, et al. Real-life comparison of beclometasone dipropionate as an extrafine- or larger-particle formulation for asthma. Respir Med 2013; 107: 987-1000.

28 Williams LK, Peterson EL, Wells K, et al. Quantifying the proportion of severe asthma exacerbations attributable to inhaled corticosteroid nonadherence. J Allergy Clin Immunol 2011; 128: 1185-1191.

29 Williams LK, Pladevall M, Xi H, et al. Relationship between adherence to inhaled corticosteroids and poor outcomes among adults with asthma. J Allergy Clin Immunol 2004; 114: 1288-1293.

30 Balkrishnan R, Christensen DB. Inhaled corticosteroid nonadherence and immediate avoidable medical events in older adults with chronic pulmonary ailments. J Asthma 2000; 37: 511-517.

31 Mattke S, Martorell F, Hong SY, et al. Anti-inflammatory medication adherence and cost and utilization of asthma care in a commercially insured population. J Asthma 2010; 47: 323-329.

32 Delea TE, Stanford RH, Hagiwara M, et al. Association between adherence with fixed dose combination fluticasone propionate/salmeterol on asthma outcomes and costs. Curr Med Res Opin 2008; 24: 3435-3442.

33 Stern L, Berman J, Lumry W, et al. Medication compliance and disease exacerbation in patients with asthma: a retrospective study of managed care data. Ann Allergy Asthma Immunol 2006; 97: 402-408.

34 McMahon AD, Lipworth BJ, Davey PG, et al. Continuity of prescribing with inhaled corticosteroids and control of asthma. Pharmacoepidemiol Drug Saf 2000; 9: 293-303.

35 Smith K, Warholak T, Armstrong E, et al. Evaluation of risk factors and health outcomes among persons with asthma. J Asthma 2009; 46: 234-237.

36 Hyland ME, Whalley B, Halpin DM, et al. Frequency of non-asthma GP visits predicts asthma exacerbations: an observational study in general practice. Prim Care Respir J 2012; 21: 405-411.

37 Osman LM, Friend JA, Legge JS, et al. Requests for repeat medication prescriptions and frequency of acute episodes in asthma patients. J Asthma 1999; 36: 449-457. 
38 Rohan J, Drotar D, McNally K, et al. Adherence to pediatric asthma treatment in economically disadvantaged African-American children and adolescents: an application of growth curve analysis. J Pediatr Psychol 2010; 35: $394-404$.

39 McNally KA, Rohan J, Schluchter M, et al. Adherence to combined montelukast and fluticasone treatment in economically disadvantaged African-American youth with asthma. J Asthma 2009; 46: 921-927.

40 Krishnan JA, Bender BG, Wamboldt FS, et al. Adherence to inhaled corticosteroids: an ancillary study of the Childhood Asthma Management Program clinical trial. J Allergy Clin Immunol 2012; 129: 112-118.

41 Lasmar L, Camargos P, Champs NS, et al. Adherence rate to inhaled corticosteroids and their impact on asthma control. Allergy 2009; 64: 784-789.

42 Santos Pde M, D’Oliveira A Jr, Noblat Lde A, et al. Preditores da adesão ao tratamento em pacientes com asma grave atendidos em um centro de referência na Bahia [Predictors of adherence to treatment in patients with severe asthma treated at a referral center in Bahia, Brazil]. J Bras Pneumol 2008; 34: 995-1002.

43 Martin BC, Wiley-Exley EK, Richards S, et al. Contrasting measures of adherence with simple drug use, medication switching, and therapeutic duplication. Ann Pharmacother 2009; 43: 36-44.

44 Desai M, Oppenheimer JJ. Medication adherence in the asthmatic child and adolescent. Curr Allergy Asthma Rep 2011; 11: 454-464

45 Boulet LP, Vervloet D, Magar Y, et al. Adherence: the goal to control asthma. Clin Chest Med 2012; 33: 405-417.

46 Riekert KA, Butz AM, Eggleston PA, et al. Caregiver-physician medication concordance and undertreatment of asthma among inner-city children. Pediatrics 2003; 111: e214-e220.

47 Krishnan JA, Riekert KA, McCoy JV, et al. Corticosteroid use after hospital discharge among high-risk adults with asthma. Am J Respir Crit Care Med 2004; 170: 1281-1285.

48 Hess LM, Raebel MA, Conner DA, et al. Measurement of adherence in pharmacy administrative databases: a proposal for standard definitions and preferred measures. Ann Pharmacother 2006; 40: 1280-1288.

49 Mattke S, Jain AK, Sloss EM, et al. Effect of disease management on prescription drug treatment: what is the right quality measure? Dis Manag 2007; 10: 91-100.

50 Steiner JF, Earnest MA. The language of medication-taking. Ann Intern Med 2000; 132: 926-930.

51 Inhaler Error Steering Committee, Price D, Bosnic-Anticevich S, et al. Inhaler competence in asthma: common errors, barriers to use and recommended solutions. Respir Med 2013; 107: 37-46.

52 Newman SP. Scintigraphic assessment of therapeutic aerosols. Crit Rev Ther Drug Carrier Syst 1993; 10: 65-109.

53 Laube BL, Janssens HM, de Jongh FH, et al. What the pulmonary specialist should know about the new inhalation therapies. Eur Respir J 2011; 37: 1308-1331.

54 Grover C, Armour C, Asperen PP, et al. Medication use in children with asthma: not a child size problem. J Asthma 2011; 48: 1085-1103.

55 Busse WW, Morgan WJ, Taggart V, et al. Asthma outcomes workshop: overview. J Allergy Clin Immunol 2012; 129: Suppl. 3, S1-S8.

56 Helms PJ. "Real world" pragmatic clinical trials: what are they and what do they tell us?. Pediatr Allergy Immunol 2002; 13: 4-9.

57 Schmier JK, Leidy NK. The complexity of treatment adherence in adults with asthma: challenges and opportunities. J Asthma 1998; 35: 455-472.

58 Bousquet J, Schünemann HJ, Zuberbier T, et al. Development and implementation of guidelines in allergic rhinitis - an ARIA-GA2LEN paper. Allergy 2010; 65: 1212-1221.

59 Dales RE, Cakmak S, Judek S, et al. Influence of outdoor aeroallergens on hospitalization for asthma in Canada. J Allergy Clin Immunol 2004; 113: 303-306.

60 Smyth AR, Barbato A, Beydon N, et al. Respiratory medicines for children: current evidence, unlicensed use and research priorities. Eur Respir J 2010; 35: 247-265.

61 Easterbrook PJ, Berlin JA, Gopalan R, et al. Publication bias in clinical research. Lancet 1991; 337: 867-872.

62 Schlender A, Alperin PE, Grossman HL, et al. Modeling the impact of increased adherence to asthma therapy. PLoS One 2012; 7: e51139.

63 Forno E, Celedón JC. Predicting asthma exacerbations in children. Curr Opin Pulm Med 2012; 18: 63-69. 